\title{
ANGULAR MOMENTUM SPINNER
}

\author{
Yizong $\mathrm{He}^{1 *}$ and Lijuan $\mathrm{You}^{2}$ \\ ${ }^{1}$ University of Minnesota, USA \\ ${ }^{2}$ Lakes International Language Academy, USA \\ *Corresponding author: yizonghe@126.com
}

\begin{abstract}
An angular momentum spinner includes two or more rotating arms loaded with an adjustable sliding block for each arm and laid symmetrically about a rotating center. Each sliding block is controlled to move along the corresponding rotating arm. Such a spinner can display the conservation of angular momentum vividly and intuitively, and can also serve as an amusing toy for children. The spinner can be used as a teaching equipment to demonstrate the theorem of angular momentum conveniently, intuitively and amusingly.
\end{abstract}

\section{KEYWORDS}

fidget spinner, angular momentum spinner, theorem of angular momentum, law of conservation of angular momentum, teaching apparatus

\section{INTRODUCTION}

In 1993, Catherine Hettinger was unable to play with her seven-year-old daughter due to myasthenia gravis, so she designed a Fidget Spinner serving as a distracting toy shown in Figure 1 [1]. The left photo in Figure 1 is a spinning fidget spinner while the right photo shows the fidget at rest. The Fidget Spinner is comprised of a ball bearing from which three rotating arms stretch symmetrically. Although Hettinger's daughter enjoyed her spinner, the Fidget Spinner was ignored for more than ten years until 2005. After 2005 small manufacturers started making various spinners with all kinds of shapes and colors, and marketed them as therapeutic aids for kids with anxiety or attention deficit, helping them relieve stress and better focus on the task at hand. Today millions of elementary to senior high school students regard fidget spinners as their favorite toys. The advantage of the fidget spinner is the simplicity of its structure, making manufacturing easy with low cost. On the other hand, the structure is so simple making it function monotonously, rotating only with decreasing speed due to the force of friction.

A common way to demonstrate the theorem of angular momentum is to rotate a man sitting on a swivel chair, who stretches out and draws back his hands holding a pair of dumbbells [2]. The revolving speed of both man and chair will change as the distance of the dumbbells with respect to the rotating center changes. This is a common way used in many universities to demonstrate the theorem of angular momentum and the law of conservation of angular momentum. Obviously, it is, in some ways, cumbersome and inconvenient. 
International Journal of Instrumentation and Control Systems (IJICS) Vol.8, No.3, July 2018

Accordingly, the present design concerns an angular momentum spinner comprised mainly of two ball bearings and two or more rotating arms with a sliding block for each arm. Such a spinner combines both the function of the existing fidget spinner and the function of regulating revolving speed with ease, which demonstrates skillfully the theorem of angular momentum and the law of conservation of angular momentum.

\section{THE STRUCTURE OF ANGULAR MOMENTUM SPINNER}

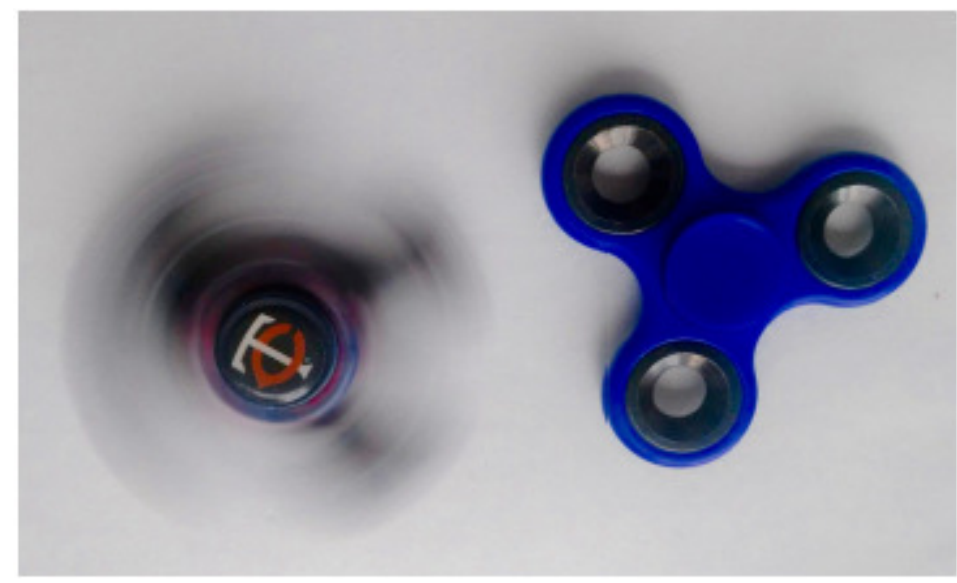

Figure 1 Existing fidget spinner

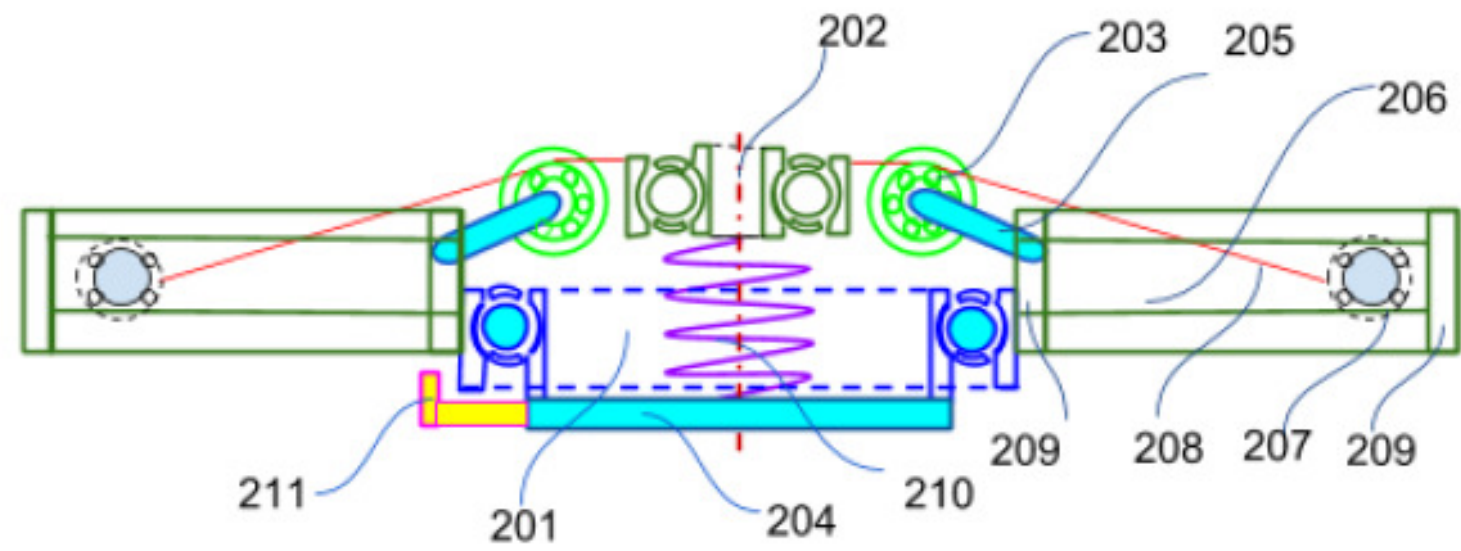

201 base ball bearing, 202 lifter ball bearing, $203 \mathrm{U}$ groove track roller, 204 cylindrical pedestal with a baffle for driving rotating arm, 205 fastening arm, 206 rotating arm, 207 sliding block, 208 steel connecting wire, 209 rectangular baffle, 210 lifter spring, 211 baffle for driving rotating arm.

Figure 2 Cross-section of an angular momentum spinner. 


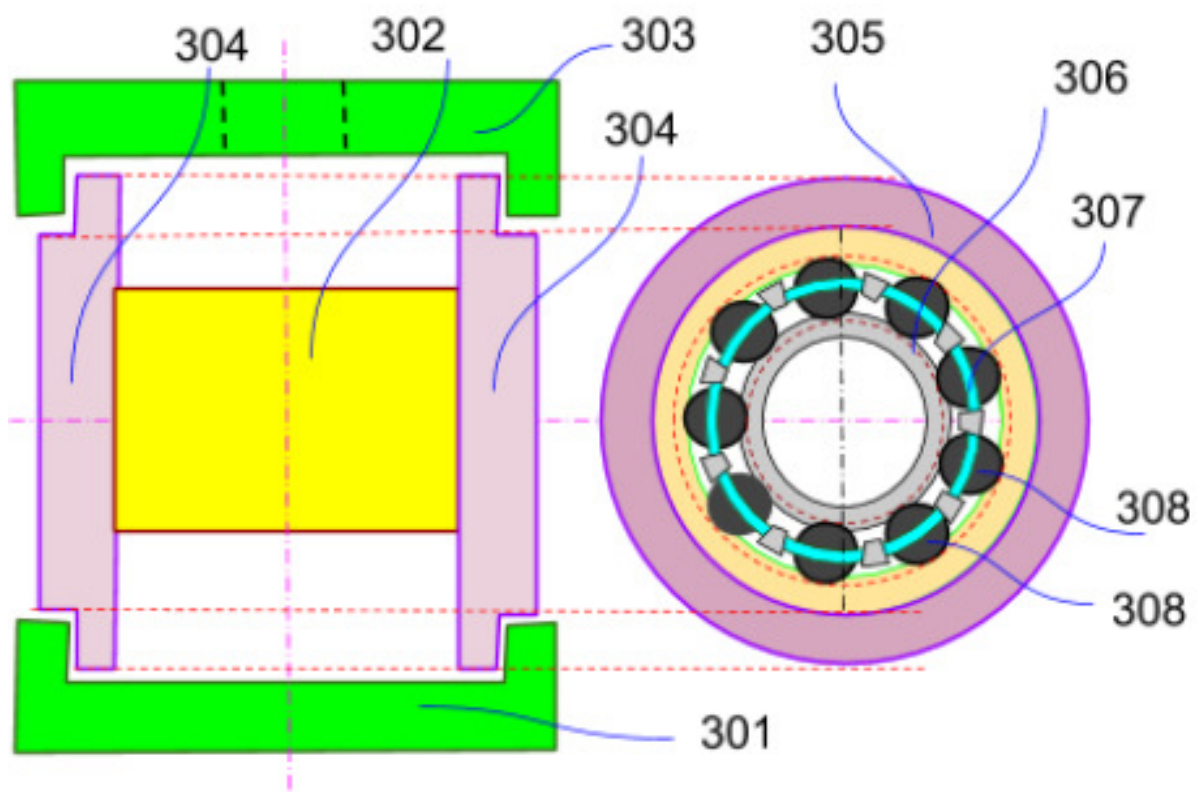

301 U-like pedestal of rotating arm, 302 cylindrical connector, 303 roof of rotating arm with a rectangular window, 304 ball bearing, 305 convex outer ring, 306 inner ring, 307 separator, 308 steel ball

Figure 3 A cross-section of the rotating arm and ball bearing
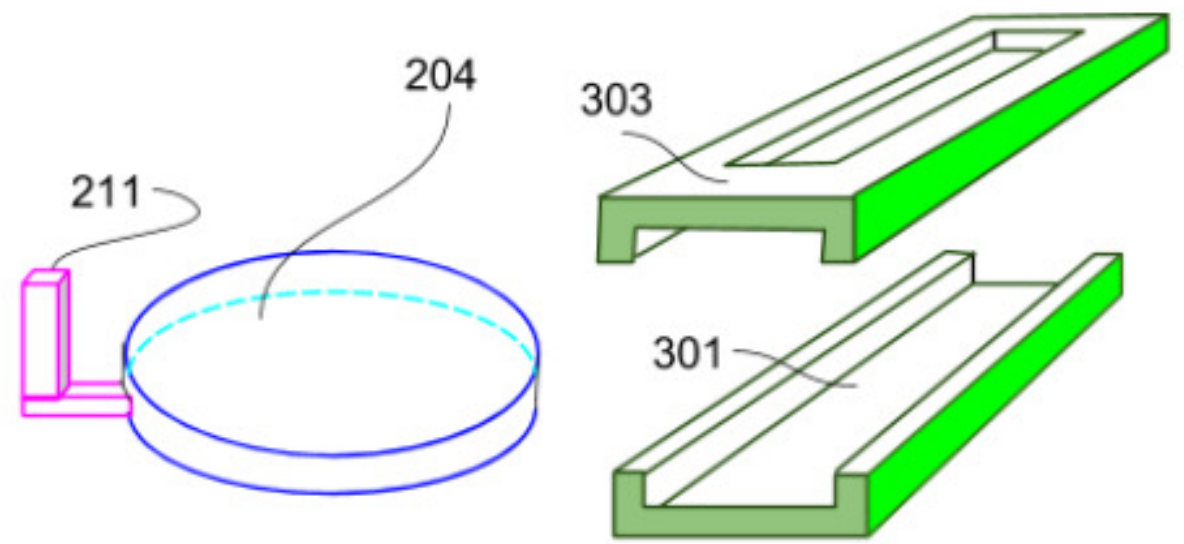

204 cylindrical pedestal with a baffle for driving rotating arm, 211 baffle for driving rotating arm, $301 \mathrm{U}$-like pedestal of rotating arm, $303 \mathrm{U}$-like roof of rotating arm with a rectangular window

Figure 4 Stereograms of the rotating arm and cylindrical pedestal with a baffle for driving the rotating arms 


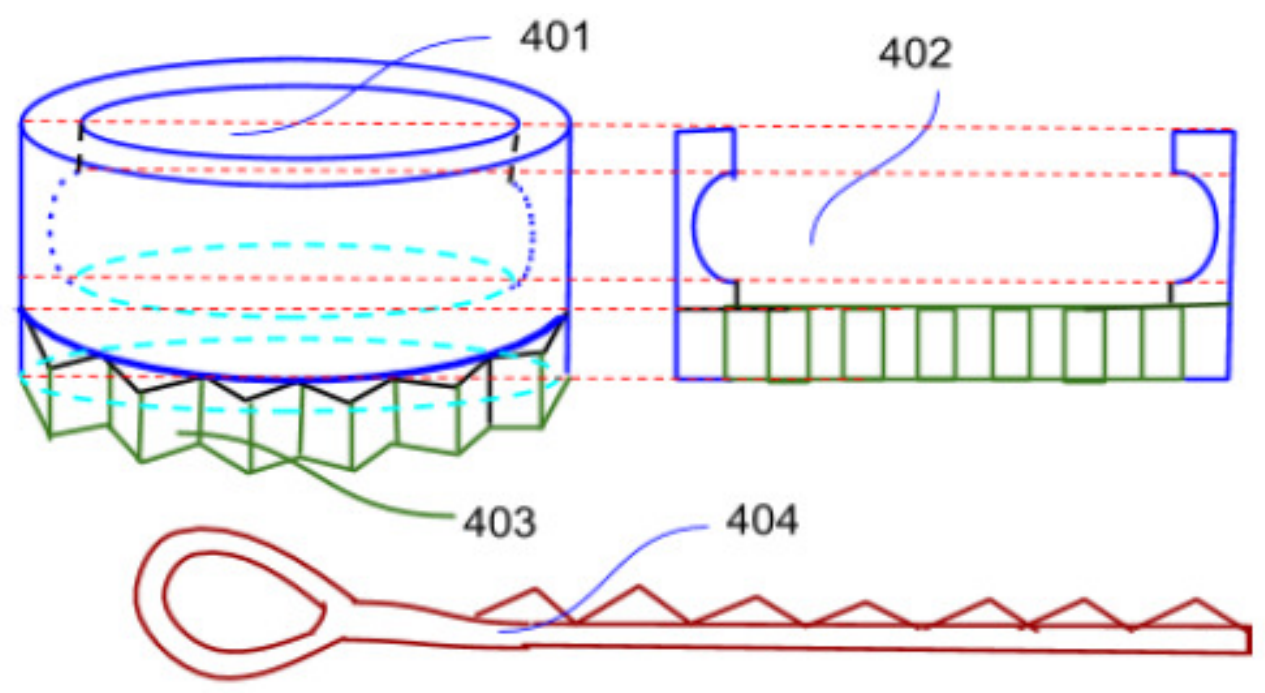

\section{1 outer ring with spline, 402 spline, 403 cross section of 401,304 soft spline}

Figure 5 Detailed picture of the outer ring of the base bearing and a soft spline to revolve the rotating arms

To achieve both functions of a fidget spinner and a teaching apparatus to demonstrate the theorem of angular momentum, the present device includes mainly two ball bearings, a U-groove track roller, rotating arms and sliding blocks. The more detailed descriptions are as following:

Figure 2 illustrates a cross-section of the overall perspective of an angular momentum spinner. Figure 3,4 and 5 are merely supplementary to Figure 2. A ball bearing is defined as small metal balls placed between the moving parts of a machine to make the parts move smoothly.

A base ball bearing 201 and lifter ball bearing 202 are laid concentrically. 202 is restrained to cylindrical pedestal 204 by lifter spring 210. A baffle is installed on the edge of 204 in order to form a gap for a soft spline to drive the rotating arms shown in Figure 5. The outer ring of 201 is connected to rigid rotating arm 206. A steel connecting wire 208 is bound to the outer ring of 202 which pulls 207 when 202 is moved downwards. 208 sits astride a U-groove track roller 203 which is connected to the rotating arm by fastening arm 205. A sliding block 207 slides back and forth on 206 by pressing and releasing 202. After the rotating arm is kicked off to revolve by the soft spline 404 (shown in Figure 5) or by hand, based on the theorem of angular momentum, all the rotating arms start to revolve and their speed can be regulated continuously by making 202 move up and down.

Figure 3 is a cross-section of the rotating arm 206 and sliding block 207 consisting of two identical ball bearings 304 connected by cylindrical connector 302. Also, the cross-section of 304 illustrates a convex outer ring 305 and inner ring 306, separator 307 and steel balls 308. In order to keep the rotating block from slipping off the track of 206, the outer ring of the ball bearing is made to be convex. Both ends of rotating arm are blocked by rectangular baffles. 
International Journal of Instrumentation and Control Systems (IJICS) Vol.8, No.3, July 2018

Pressing and releasing 202, the sliding block moves back and forth along the pedestal of rotating arm 206 between the two rectangular baffles 209. A rectangular window is carved out from the roof of rotating arm 302 so that the connecting wire 208 can connect 207 and outer ring 202.

Figure 4 shows a perspective view of parts 301 and 303 of the rotating arm of the spinner of Fig. 2. and also shows a perspective view of the cylindrical pedestal of the spinner with a post to limit the range of motion of a soft spline to drive rotating arms.

Figure 5 illustrates the structure of outer ring 401, 402 with a spline 403 for revolving the rotating arm by pulling a soft spline 404 quickly which goes through the gap between outer ring of 201 and a baffle 211 .

\section{Illustrate The Principle OF Angular Momentum}

An angular momentum spinner serves as both a toy for entertaining students and as a teaching apparatus to demonstrate the theorem of angular momentum at universities.

It is necessary to introduce the theorem of angular momentum in order to appreciate the principle of regulating revolving speed. Consider a particle of mass $\mathrm{m}$, moving with velocity vector $\mathbf{v}$ relative to some inertial frame. The origin of the coordinate frame is denoted $\mathrm{O}$. The particle has the momentum $\mathrm{m} v$ relative to the inertial frame. We define the angular momentum $\mathbf{L}$ relative to $\mathrm{O}$ as $\mathbf{r} \times$ mv. The cross symbol $\times$ between two vectors describes the vector cross product. The product $\mathbf{r} \times \mathbf{F}$ is called the torque on the particle with respect to the point $\mathrm{O}$. Based on the above, the theorem states that the rate of change of the angular momentum of a particle around some point $\mathrm{O}$ equals the torque on the particle, with respect to $\mathrm{O}$ : namely $\mathrm{d}(\mathbf{r} \times \mathrm{mv}) / \mathrm{dt}=\mathbf{r} \times \mathbf{F}$. If the net external torque on the particle is zero, then $\mathrm{d}(\mathrm{r} \times \mathrm{m} v \mathrm{v}) / \mathrm{dt}=0$. Integrating both sides gets $\mathbf{r} \times$ $\mathrm{mv}=\mathbf{C}$. Thus the angular momentum of a particle is conserved if and only if the net external torque acting on a particle is zero, namely the law of conservation of angular momentum.

The principle of regulating revolving speed can be described by the following formulas: exert force $\mathbf{F}$ to the lifter ball bearing 202, $\mathbf{r} \times \mathbf{F}=0$, because the direction of radius vector and force $\mathbf{F}$ are always either parallel or antiparallel. Therefore, the motion of the sliding block obeys the law of conservation of angular momentum $m v_{2} r_{2}-m v_{1} r_{1}=0$, where $m v_{2} r_{2}=\left|\mathbf{r}_{2} \times m \mathbf{v}_{2}\right|$ and $m v_{1} r_{1}=$ $\left|\mathbf{r}_{1} \times \mathrm{mv} 1\right|$. The relationship between net force $\mathbf{F}$ to press 202, work done to sliding block, rotating speed $\mathrm{v}$ of sliding block and the radius $\mathrm{r}$ can be express as following equations:

$$
\begin{gathered}
m v_{2} r_{2}=m v_{1} r_{1} \\
\int_{r_{1}}^{r_{2}} \mathbf{F} \cdot \mathrm{d} \mathbf{r}=\frac{1}{2} m v_{2}^{2}-\frac{1}{2} m v_{1}^{2} \\
W \text { ork }=\frac{1}{2} m v_{2}^{2}-\frac{1}{2} m v_{1}^{2}=\frac{1}{2} m v_{1}^{2}\left[\left(r_{1}^{2} / r_{2}^{2}\right)-1\right]>0
\end{gathered}
$$

where the sliding block moves from initial position $r_{1}$ to final $r_{2}\left(r_{1}>r_{2}\right)$, When the activator stops pressing 202, $\mathbf{F}=0,202$ goes up to initial position due to the lifter spring action and the centrifugal force of sliding block. Pressing and then releasing causes the sliding block to move back and forth until the rotating speed decreases to zero due to the small friction force. 
International Journal of Instrumentation and Control Systems (IJICS) Vol.8, No.3, July 2018

After the rotating arms are driven to move, the revolving motion can last for a period of time, because of the small friction force due to the use of a ball bearing. Pressing the lifter ball bearing down causes the revolving speed to go up accordingly. Convervely, ceasing to press the lifter ball bearing makes the revolving radius increase, resulting in the revolving speed decreasing linearly, obeying the law of conservation of angular momentum.

\section{DISCUSSION}

Generally speaking, moving objects easily attract a human being's attention, such as the blinking from turn signals on vehicles, or the waving one's hand in crowd are all easy to spotted. Based on continual and fast movement, the fidget spinner has become a favorite toy for most teenage students

What characterizes a fidget spinner is its structural simplicity and its movement. The structure made manufacturing easy, while the movement properties made it fascinating. By comparison, the process to manufacture an angular momentum spinner is more complex to some extent. However, the function which allows a person to regulate the spinner speed lexturely increases its interest considerably. What is more, the angular momentum spinner can serve as a good teaching apparatus to demonstrate both the laws of angular momentum and conservation of angular momentum. A good teaching tool is essential for illustrating the abstruse scientific theory. The number of rotating arms could vary from two to many more. Also, the size could range from the same size as current fidget spinners to much larger for use in universities. In a word, an angular momentum spinner surely serves as an amusing toy and useful teaching tool.

\section{REFERENCES}

[1] https://en.wikipedia.org/wiki/Fidget_spinner

[2] https://en.wikipedia.org/wiki/Angular_momentum 